\title{
Wear of a dental composite in an artificial oral environment: a clinical correlation
}

\author{
Ralph DeLong, ${ }^{1}$ Maria R. Pintado, ${ }^{1}$ William H. Douglas, ${ }^{1}$ Alex S. Fok, ${ }^{1}$ Aldridge D. Wilder, Jr., ${ }^{2}$ \\ Edward J. Swift, Jr., ${ }^{2}$ Stephen C. Bayne ${ }^{3}$ \\ ${ }^{1}$ Minnesota Dental Research Center for Biomaterials and Biomechanics, University of Minnesota School of Dentistry, \\ Minneapolis, Minnesota \\ ${ }^{2}$ Department of Operative Dentistry, School of Dentistry, University of North Carolina, North Carolina \\ ${ }^{3}$ Department Cardiology, Restorative Sciences, and Endodontics, University of Michigan School of Dentistry, \\ Ann Arbor, Michigan
}

Received 1 December 2011; revised 17 July 2012; accepted 19 July 2012

Published online 21 September 2012 in Wiley Online Library (wileyonlinelibrary.com). DOI: 10.1002/jbm.b.32801

\begin{abstract}
The study objective was to correlate wear between an in vitro method for simulating wear and in vivo wear of a posterior dental composite. Ten subjects (12 restorations) were selected from a five-year clinical study (University of North Carolina, School of Dentistry) that assessed wear of SureFil composite (Caulk, Dentsply). Subject casts were digitized and changes in volume and mean depth with time were calculated from the 3D digital models for contact and contactfree wear. SureFil composite disks were mounted in the University of Minnesota's Artificial Oral Environment, opposed by natural enamel, subjected to mandibular-like movements for $150 \mathrm{~K}, 300 \mathrm{~K}, 600 \mathrm{~K}, 1.2 \mathrm{M}$, and $1.5 \mathrm{M}$ cycles, and loaded with peak forces of $13 \mathrm{~N}(n=7)$ or $30 \mathrm{~N}(n=3)$. Wear rates were calculated as the slope of the linear regressions fitting the wear data. Data were analyzed using one-way ANOVAs and post hoc $t$-tests where appropriate $(p=0.05)$. Clinical restora-
\end{abstract}

tions included contact wear on seven restorations and contactfree wear on all restorations. Contact-free wear was less than contact wear $(p<0.01)$. SureFil clinical wear rates were 0.012 $\mathrm{mm} /$ year (mean depth) and $0.023 \mathrm{~mm}^{3} /$ year (volume). Clinical restorations expanded slightly during the first year. Using a conversion rate of one year equals $3 \times 10^{5}$ cycles, there were no significant differences between the clinical and simulated data except depths at Year 5 and $13 \mathrm{~N}$ volume at Year 4. The $30 \mathrm{~N}$ simulation reproduced the clinical data if contact-free wear was taken into account. Good agreement between simulated and clinical wear implies that in vitro simulation can screen new composite formulations. (๑) 2012 Wiley Periodicals, Inc. J Biomed Mater Res Part B: Appl Biomater 100B: 2297-2306, 2012.

Key Words: wear, tribology, test method, dental composite, simulated wear

How to cite this article: DeLong R, Pintado MR, Douglas WH, Fok AS, Wilder AD Jr., Swift EJ Jr., Bayne SC. 2012. Wear of a dental composite in an artificial oral environment: a clinical correlation. J Biomed Mater Res Part B 2012:100B:2297-2306.

\section{INTRODUCTION}

US Private practitioners placed 122.7 million dental resin composites in 2006. ${ }^{1}$ Longevity and survival studies have shown an average replacement time of 5.7 years for dental resin composites. ${ }^{1}$ Many factors contribute to premature failure including improper handling of materials, ineffective bonding system placement, incomplete composite curing, improper occlusal adjustments, ineffective finishing and polishing steps, postoperative sensitivity, marginal staining that often mimics secondary caries, and occlusal wear. Development of longer-lasting composite restorations is an urgent dental and orofacial health need. Use of such new materials would have a significant positive impact on the oral health of the US population.

Development of improved composite restorations requires clinical and laboratory evaluative techniques to permit expedited assessment of clinical properties. One very important clinical property is wear. ${ }^{2}$ Today's composites are wear resistant. Minor modifications in composite formulation can cause dramatic changes in wear performance. Therefore, newly developed dental materials should be thoroughly assessed for performance under the repetitive loads experienced in the oral environment. An effective laboratory simulation that reproduced the oral biomechanics would be of widespread value in shortening the time span for the chairside delivery of new dental materials provided it correlated with observed clinical outcomes.

Different occlusions, restoration sizes, and locations can affect service life of a restorative. ${ }^{3}$ These factors lead to wide variations in reported clinical service life of restorative materials. Service life is further complicated by the different wear mechanisms that the restorative material experiences in the oral cavity (attrition, abrasion, erosion, fracture, and fatigue). Occlusal wear is categorized as contact wear in areas experiencing tooth-to-tooth contacts during occlusion 
Clinical Wear Study (In Vivo)

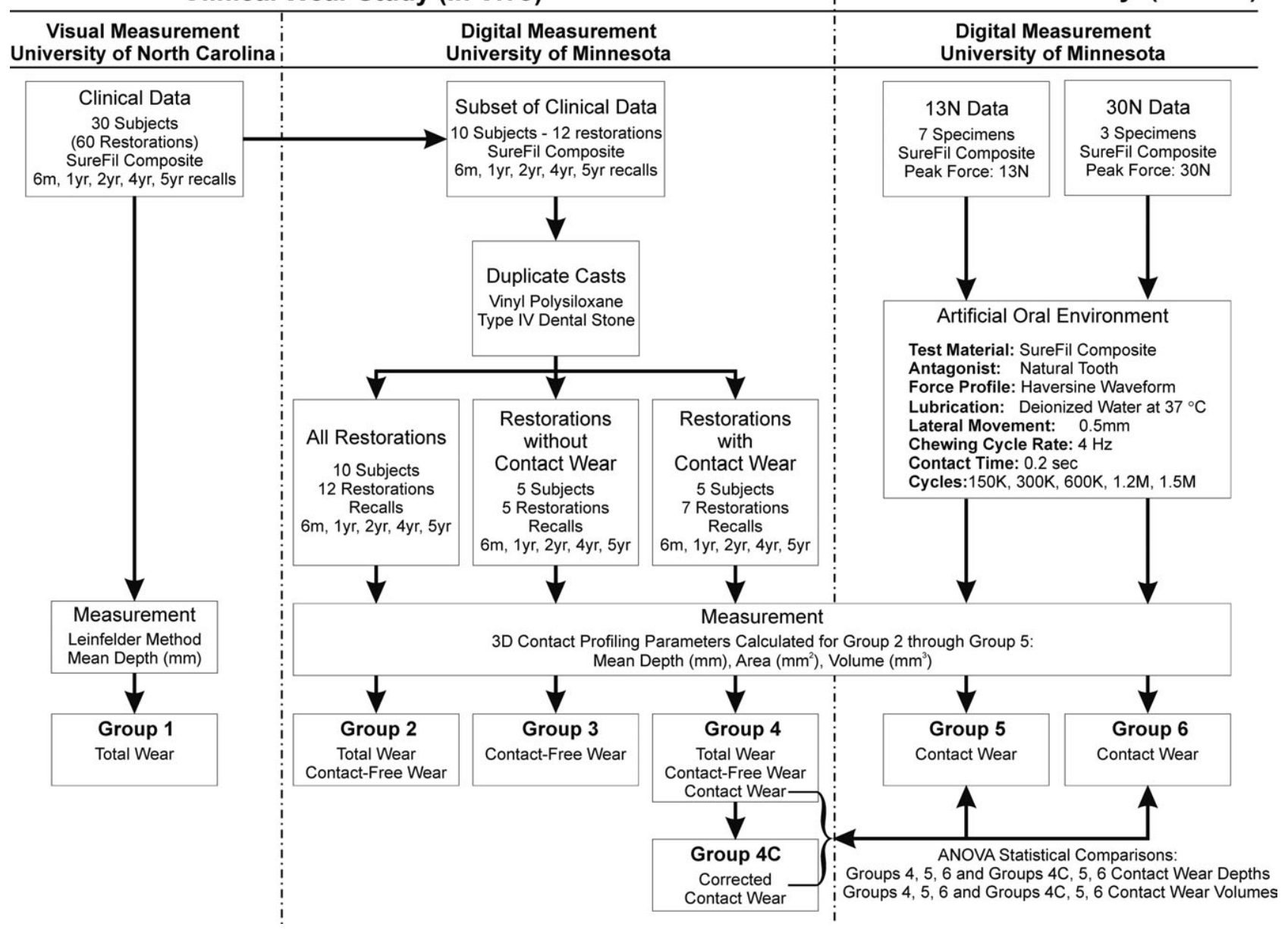

FIGURE 1. Study design.

Simulated Wear Study (In Vitro)
13 N Data

7 Specimens
Artificial Oral Environment Antagonist: Natural Tooth

Contact Wear $\quad$ Contact Wear

5 Subjects Restoration Recalls 5 Restoration

Measurement orce Profile: Haversine Waveform and contact-free wear in areas subjected to abrasion from hard particles in the food bolus.

Clinical data used to validate simulated wear must meet three criteria. First, it must be from a high quality clinical study of at least three years duration to account for wear caused by fatigue. Second, results should be reported in a format consistent with that used in the simulation. Preferably, accurate clinical replicas would be available so that wear could be measured using the same methodology. Third, the same material formulation used in the clinical study must be available for the simulation. Due to frequent changes in commercial composite compositions, some dental materials are no longer available for in vitro correlation.

The objective of this study was to correlate wear parameters for wear patterns between an in vitro method for simulating wear and in vivo wear of a posterior dental composite material. The in vivo assessment employed both visual and digital methods to measure wear. The in vitro study assessment used only digital methods.

\section{MATERIALS AND METHODS}

In vivo and in vitro methodologies were carried out on the same composite (SureFil; Caulk, Dentsply) (Figure 1). Sure-
Fil, a packable composite for use in posterior teeth, showed good early properties in both laboratory studies and two clinical trials. ${ }^{4-6}$ The current study is a collaboration with researchers who completed a 5-year clinical trial at the University of North Carolina. This study met the three criteria necessary for a correlation between in vitro and in vivo wear.

\section{Clinical study-University of North Carolina}

The clinical trial carried out at the University of North Carolina (UNC), School of Dentistry has been reported by Bayne et al. ${ }^{7}$ The study comprised 30 patients $(62 \%$ women; mean age: 42 years). Restorations were placed primarily as amalgam replacements. Cavity preparations (unbeveled enamel margins, moist dentin) were bonded on enamel and dentin with two separate coats of Prime\&Bond NT (Caulk/Dentsply; Ver. 2.1, Lot 9707261) with gentle air drying (5 s) and light curing (10 s). Materials (SureFil, Batches = 34297, $847813,545112,9707261$ ) were placed in two increments and cured separately for $40 \mathrm{~s}$. Finishing and polishing were conducted with Enhance points/cups/disks (Caulk/Dentsply), followed by Prisma Gloss and Prisma Gloss X-Fine pastes (Caulk/Dentsply). Impressions of the restorations 


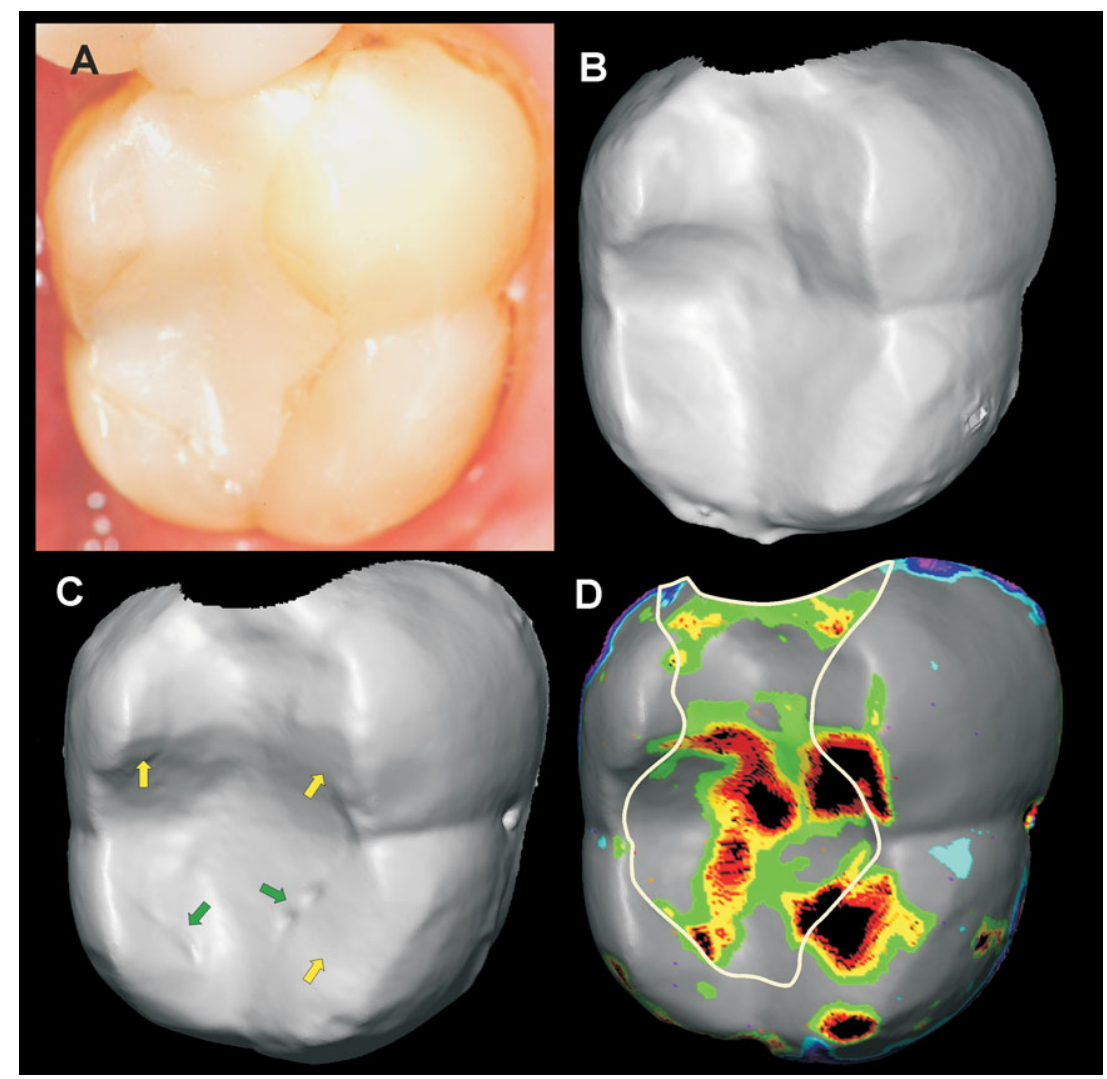

FIGURE 2. Clinical analysis of wear: A) 5 Year clinical photograph of restoration. B) Digital image of restoration at baseline. C) Digital image of restoration at 5 years. The orange arrows indicate contact wear on enamel. The green arrows show areas where the margin of the restoration has fractured. Fractures were not included in the wear calculations. D) A difference plot of the 5 year image subtracted from the baseline image. The cream colored line is the outline of the restoration. Scale: Gray $=+/-0.025 \mathrm{~mm}$; green $=-0.025$ to $-0.050 \mathrm{~mm}$; yellow $=-0.050$ to $-0.075 \mathrm{~mm}$; orange $=-0.075$ to $-0.100 \mathrm{~mm}$, red $=-0.100$ to -0.125 ; black $>0.125 \mathrm{~mm}$. Light blue, blue, purple, and violet are similar, but with a positive sign. A minus sign means that material was lost. [Color figure can be viewed in the online issue, which is available at wileyonlinelibrary.com.]

were made at $0.5,1,2,4$, and 5 years time intervals using vinyl polysiloxane impression material (Aquasil, Caulk/ Dentsply). Replicas were made using ResinRock stone (Whip Mix). Mean depth loss was assessed visually using the Leinfelder method. ${ }^{8}$ From the 30 subjects, 15 possibilities were selected that had all recall datasets. The UNC group selected a subset of casts (10 subjects, 12 restorations) based on tooth type (first or second molar) and restoration type (Class 1 or Class 2 restorations with facial or lingual extensions). The casts were sent to the University of Minnesota for further evaluation.

\section{Clinical study-University of Minnesota}

Casts were occlusally mapped using the University of Minnesota (UMN) contact profiling system, ${ }^{9}$ with $\mathrm{X}, \mathrm{Y}$, and Z resolutions of $0.050 ; 0.100 ; 0.001 \mathrm{~mm}$ respectively, and a point accuracy of $\pm 0.007 \mathrm{~mm}$. Avoid possible scratching of the original clinical casts by the profiler stylus, each cast was duplicated using a vinyl polysiloxane impression material (Imprint II Garant Light Body, 3M ESPE, St Paul, MN) in a Kwik-Tray metal quadrant tray (Kerr Brand, Emeryville, CA). Replica casts were made from the impressions using a type IV dental stone (Fujirock EP; GC Europe, Leuven, Belgium).
Graphic renditions of the baseline and worn specimens were superimposed using a least squared fit, and differences were identified using AnSur Software (Copyright Regents University of Minnesota) (Figure 2). Restoration margins were identified from the 3D surface images and verified using 5-year clinical photographs provide by the UNC group (Figure 2A). Changes in surface anatomy with time were calculated for mean depth, projected area, and volume loss for all sources of wear due to masticatory function and parafunction over the restoration surface (Group 1). Depth was calculated by averaging the difference in surface topologies for restorations before and after wear in a direction perpendicular to the occlusal plane. Area was calculated as the topological surface projected onto the occlusal plane. Volume was calculated as the volume of material between the surface topologies for restorations before and after wear.

Opposing casts and information on occlusal contacts were not available; therefore, interrogation of the superimposed digital images was used to separate contact and contact-free wear. For 5 of the 10 casts ( 7 restorations), clearly defined contact wear facets were identified as regions of localized surface degradation not attributable to fracture or artifacts (Figure 2C). ${ }^{10}$ Similarly, in the same teeth, occlusal 
contact-free areas could be identified where there were no occlusal contacts, fractures, or artifacts (bubbles, saliva, etc.). This differentiation enabled the separation of the occlusal contact (Group 4) and contact-free (Group 3) areas on these restorations. In the five remaining casts (five restorations), the contact facets could not be differentiated with certainty; therefore, they were not included in the calculation (Group 2). Fractures were identified as well-defined regions on a restoration that displayed an abrupt change in surface topology present in all subsequent recall specimens (Figure 2C). Loss from fracture was excluded from the calculations.

For comparison with the simulated wear, it was assumed that clinical contact-free wear occurred in the contact wear area; therefore, clinical contact mean depths and volumes were corrected using the contact-free data (Group 4C). For each restoration exhibiting contact wear, contact depth was "corrected" by subtracting the nominal depth, which was calculated as contact-free volume divided by contact-free area. Contact volume loss was "corrected" by subtracting a contact-free volume loss calculated as the contact wear projected area times the nominal depth.

\section{Laboratory study-University of Minnesota}

Seven SureFil composite disks (Lot 060117), 12.5 mm diameter and $2 \mathrm{~mm}$ thick were prepared following manufacturer's directions, and stored for $24 \mathrm{~h}$ at $37^{\circ} \mathrm{C}$ in deionized water. Composite was packed in an aluminum mold placed on a glass slide, then a second glass slide was placed on top. The slides were clamped together with light pressure. The composite was cured $40 \mathrm{~s}$ on both sides. Samples were removed from the mold and polished on one side using medium, fine, and superfine 3M Sof-Lex disks. Each disc was mounted in UMN's Artificial Oral Environment ${ }^{11}$ and opposed by a natural enamel third molar centric cusp (Figure 3). Molars were selected from a bank of teeth stored in refrigerated deionized water with Thymol added as an antibacterial agent.

Using the physiological measurements of Ahlgren and OWall, ${ }^{12}$ a mandibular-like movement was generated with a $0.5 \mathrm{~mm}$ lateral movement and a haversine force profile with a peak force of $13 \mathrm{~N}$ and contact duration of $0.2 \mathrm{~s}$. Deionized water circulating at $37^{\circ} \mathrm{C}$ provided lubrication. Wear was calculated following $150 \mathrm{~K}, 300 \mathrm{~K}, 600 \mathrm{~K}, 1.2 \mathrm{M}$, and $1.5 \mathrm{M}$ cycles using the same mapping technique used for the clinical specimens ( $300 \mathrm{~K}$ cycles was presumed to represent 1 year of clinical wear; Group 5). The laboratory experiment was repeated with three additional discs using a peak occlusal force of $30 \mathrm{~N}$ (Group 6). Occlusal contact depth and volume wear of the artificial environment was compared to the clinical contact depth and volume wear.

\section{Statistical analysis}

Regression analysis of the depth and volume data used Microsoft Office Excel 2003. Statistical comparisons were completed for the contact wear of Group 4 with the contactfree wear of Groups 2 and 3 and the contact wear of Groups 5 and 6, and for Groups 4C, 5, and 6. Individual time points

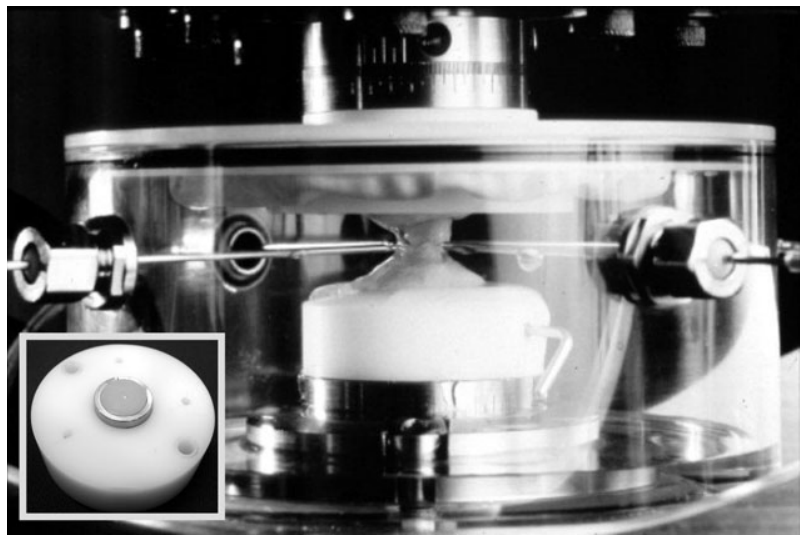

FIGURE 3. Artificial oral environment test chamber. A natural maxillary third molar was mounted on the upper fixed element of the test machine. A test specimen, shown in the insert, is mounted on the lower moveable element. The lower element moves buccal-lingually under stroke control and vertically under load or stroke control. A mode switch is made from stroke to load control just prior to contact between the opposing enamel and composite test specimens. Deionized water at $37^{\circ} \mathrm{C}$ is continuously injected into the interface between the opposing surfaces. The chamber image, which is from a different study, shows opposing maxillary and mandibular teeth.

were evaluated separately using one-way ANOVAs ( $p=$ 0.05). Post hoc analyses, where appropriate, were done using a $t$-test. SAS v9.1.3 was used for the analyses. Group 1 was not compared statistically to Groups 2, 3, and 4 because they are subgroups of Group 1. Comparisons were made for wear depth and volume losses. Separate repeated measures models compared Group 4 and corrected Group 4C to Groups 5 and 6 across the time points. "Group" and "Time" were treated as fixed effects and the within-correlation was modeled using a first-order autoregressive covariance structure (i.e., measures closer in time are more correlated). The interaction between group and time was also tested.

\section{Calculation error}

Reported values are means and standard deviations for each time interval. The standard deviations include both biological and methodological variability. The biological difference between restorations results from differences in size, location and masticatory factors. Methodological differences depend on the accuracies of the replicas, their 3D digital images, and the alignment of the baseline and recall images. Methodological errors (difference from the "true" value) are approximately $0.025 \mathrm{~mm}$ or better over the entire functional area of the tooth. ${ }^{13}$ Methodological error primarily depends on impression accuracy. Because methodological differences are much smaller than biological differences the standard deviations mainly represent biological variation.

\section{Sample size}

In vitro simulations require significantly fewer samples than clinical studies because the biological variation present in clinical studies is not present in the simulation. The only biological component in the simulation is the opposing natural tooth; all other factors are held constant. Each specimen 
required five continuous days in the artificial oral environment to complete the $1.5 \mathrm{M}$ cycles. While this was significantly less time than for the parallel clinical study, it was large enough to demand efficiency in design for the number of specimens being evaluated. The number of samples for the $13 \mathrm{~N}$ tests was set equal to the number of restorations with contact wear. An additional three samples were tested using a $30 \mathrm{~N}$ force. Because each sample required one week of machine time and because the Coefficients of Variation for Years 4 and 5 were less than 15\%, no additional samples were run.

\section{RESULTS}

Wear parameters were calculated for in vivo total wear (Group 1), contact-free wear (Groups 2 and 3) and contact wear (Group 4) and in vitro contact wear (Groups 5 and 6) (Table I). Total in vivo wear in terms of mean depth for the subset of 12 restorations (Group 1) was similar to that reported by Bayne et al. $^{7}$ for the total set of restorations except at Year 4 (Figure 4). Mean depth for In vivo contact wear (Group 4) was consistently larger than that for the in vivo total wear at all time points (Figure 4).

Group 2 contained one outlier that was consistently more than two standard deviations below the mean. The restoration was unusual in that SureFil was place against an amalgam restoration. With or without the outlier, there was no significant difference in the contact-free wear of Groups 2 and $3(p>0.05)$; therefore, these groups were pooled. The in vivo contact-free wear (Groups 2 and 3) differed significantly from the in vivo contact wear (Group 4; $p<0.01$ ). Examination of the combined contact-free data (Groups 2 and 3; Figure 5) shows a distinct nonlinearity during the first two years. The negative intercept implies the restoration increased in size.

Contact wear rates, calculated as the linear regression slopes, were all significant $(p<0.01)$ with correlation coefficients ranging from $r^{2}=0.20$ to $r^{2}=0.94$ (Table II). In vivo contact wear rates were compared with corresponding in vitro wear rates to calculate the number of simulated cycles that corresponds to one year of clinical wear. Conversion factors ranged from 1 to $3 \times 10^{5}$ cycles for mean depth and from 3.2 to $8.2 \times 10^{5}$ cycles for volume (Table III). The in vivo and in vitro contact depth and volume data were plotted using the conversion rate of $3 \times 10^{5}$ cycles equal to 1 year (Figures 6 and 7) and the Group 4C conversion factors of Table III (Figures 8 and 9).

Using a conversion rate of one year equals $3 \times 10^{5}$ cycles, repeated measures models across the time points found no statistically significant group differences comparing clinical group (Group 4) to the $13 \mathrm{~N}$ (Group 5) and 30 $\mathrm{N}$ (Group 6) for group depths $(p=0.979)$ and volumes ( $p$ $=0.347)$ or the corrected Group 4C and simulated group depths $(p=0.453)$ and volumes $(p=0.429)$. Visually (Figure 6), the corrected clinical mean depth showed good agreement with mean depths for simulated $13 \mathrm{~N}$ (Group 5) and $30 \mathrm{~N}$ (Group 6) wear during the first two years; however, it underestimated the depths in Years 4 and 5. Running one-way ANOVAs at specific time points, differences between corrected and simulated mean depths at Year 5 were barely significant $(p=0.045)$. A post hoc analysis revealed differences between the corrected clinical depths and the simulated $13 \mathrm{~N}$ depths $(p=0.046)$ and $30 \mathrm{~N}$ depths $(p=0.028)$. Contact wear measured by volume of material removed was similar for clinical Group 4 and simulated Group 6 (Figure 7) through the first two years of the clinical study. In Years 4 and 5, the clinical contact volume wear (Group 4) was greater than the simulated contact volume wear. Differences between the measured clinical and simulated volumes at Year 4 were slightly significant ( $p=$ 0.039). A post hoc analysis showed only the difference between the measured clinical volumes and simulated $13 \mathrm{~N}$ volumes was significant $(p=0.012)$. The corrected contact volume (Group 4C) was not significantly different from the $30 \mathrm{~N}$ simulated contact volume wear (Group 6) (Figure 7). There were no significant group-by-time interactions $(p>$ $0.05)$.

Contact areas behaved in a manner similar to volume wear (Table I). Contact areas increased with time for Groups 4,5 , and 6 . There was good agreement between the contact areas of Groups 4 and 6 for the first two years. Clinical contact areas (Group 4) were larger in Years 4 and 5. Group 5 wear areas were consistently smaller than those of Groups 4 and 6. Contact-free areas for Group 3, which included restorations with contact wear areas, tended to decrease with time as the contact areas increased. The contact-free areas for Group 2, which included restorations without contact wear areas, remained relatively constant with time.

\section{DISCUSSION}

The objective of a laboratory simulation is to produce wear that correlates well with clinical performance and that can predict survival time. Clinical wear is often reported as loss of material with time. The classical wear equation ${ }^{14}$ can be written as a function of time:

$$
\frac{V}{t}=\frac{K \times W_{t}}{H}
$$

where $V$ is the volume of material removed, $H$ is the hardness pressure of the wearing material, $K$ is a constant that depends on the opposing materials and experimental conditions, $t$ is time, and $W_{t}$ is the work done per unit of time. $W_{t}$, which is the product of force $(F)$ times the sliding distance per unit time $\left(L_{t}\right)$, is independent of the material being tested. Clinical studies measuring wear assume that occlusal forces and contact paths, which are highly variable both within and between subjects, ${ }^{15}$ can be represented by average values that remain relatively stable with time; thus, the clinical work done should be constant with time. If $W_{t}$, $K$, and $H$ are all constant, then volume loss with time is constant. This linear relationship has been demonstrated both in vitro, ${ }^{16}$ in vivo ${ }^{17}$ and in this study. Although the regressions lines for the in vivo and in vitro volume data (Table II) did not pass through zero as predicted by a linear relationship, their offsets, with the exception of the $30 \mathrm{~N}$ in vitro data $(p=0.046)$, were not significantly different from zero. 


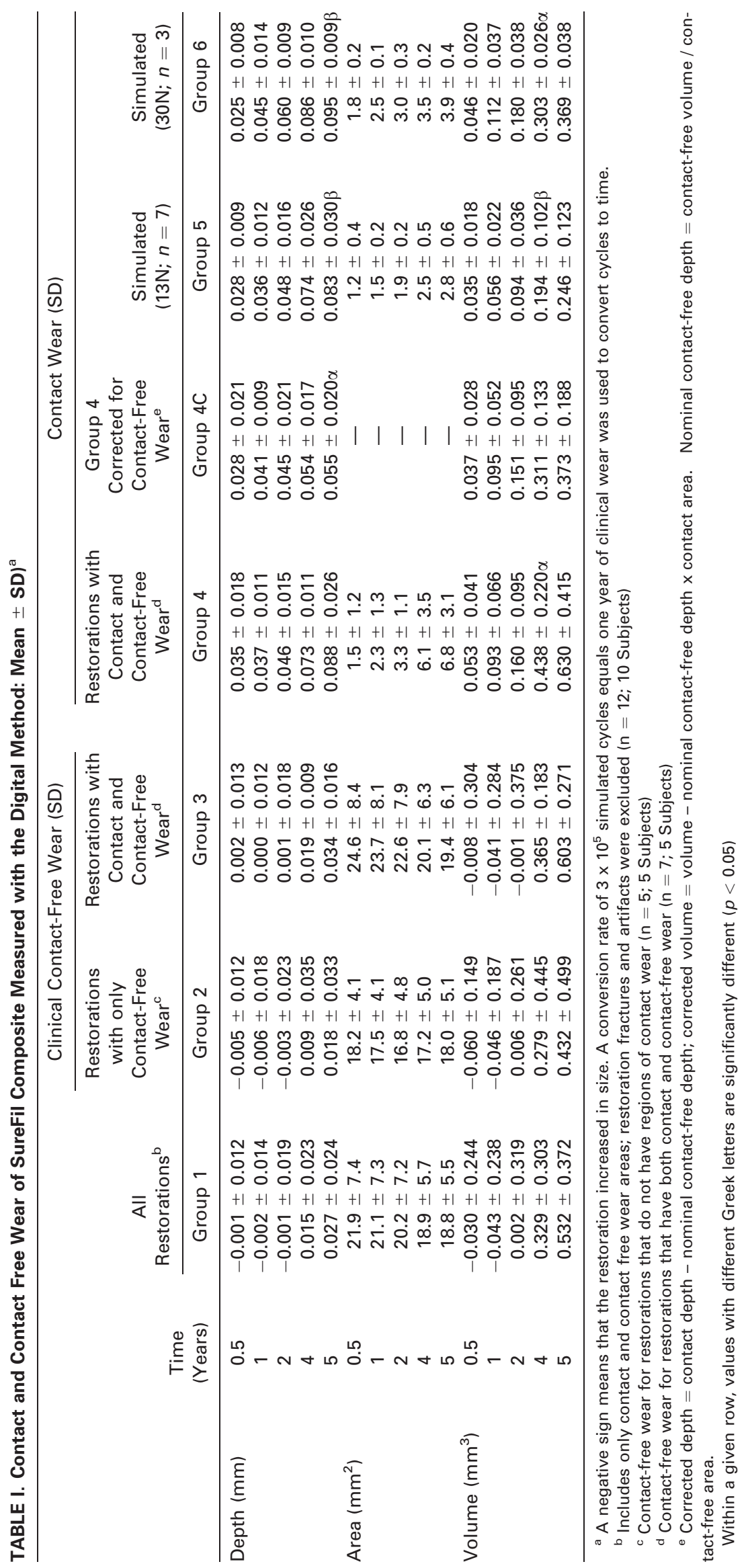




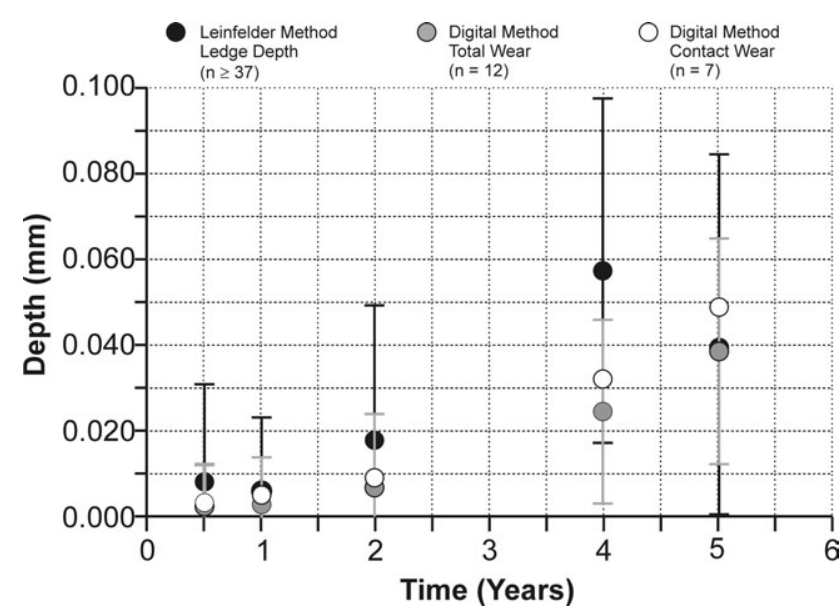

FIGURE 4. Clinical data: Leinfelder Method versus Digital Total Wear Mean Depth. Total wear includes contact and contact-free wear. Fractures are not included in the wear assessment. The error bars represent the standard deviations.

The linear relationship supports the assumption that the clinical work done, $K$, and $H$ are relatively constant with time.

To predict long term durability of the restoration, in vitro cycles must be converted to clinic time. Dividing the material loss in the clinic by the material loss in the simulation and rearranging terms:

$$
t_{\text {Clinic }}=\frac{C_{\text {Simulation }}}{C_{\text {Clinic }}} \times \frac{V_{\text {Clinic }}}{V_{\text {Simulation }}} \times t_{\text {Simulation }}
$$

where

$$
\frac{C_{\text {Simulation }}}{C_{\text {Clinic }}}=\frac{\left(K \times W_{t}\right)_{\text {Simulation }} \times H_{\text {Clinic }}}{\left(K \times W_{t}\right)_{\text {Clinic }} \times H_{\text {Simulation }}}
$$

It is reasonable to assume that clinical and simulation values of $H$ are similar. The $K_{\mathrm{s}}$, which are determined by the opposing materials and the test conditions, and the clinic work, $\left(W_{t}\right)_{\text {clinic, }}$ are unknown. The simulation work, $\left(W_{t}\right)_{\text {simu- }}$ lation, is known and adjustable. If the testing conditions and materials used in the simulation are the same as the clinical situation, $C_{\text {simulation }}=C_{\text {clinic; }}$ then:

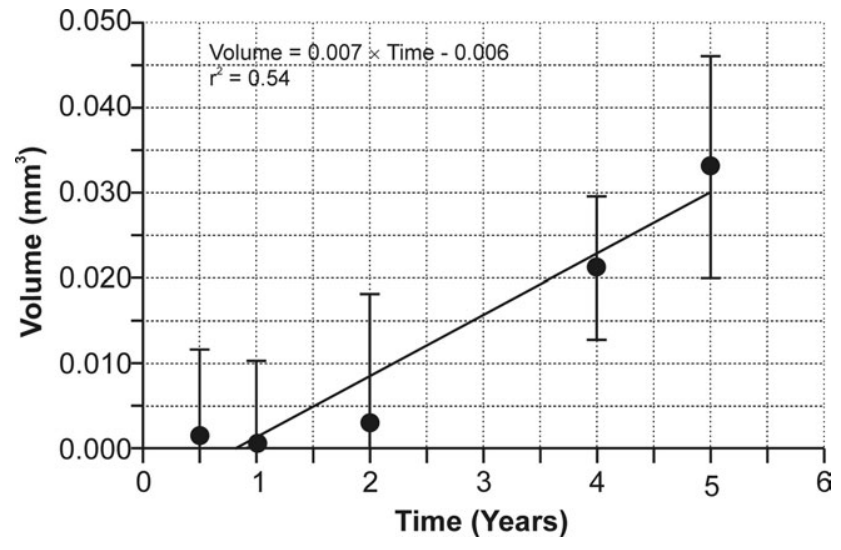

FIGURE 5. Clinical contact-free volume loss. One outlier, which was consistently two standard deviations below the mean at all time points, was excluded. Expansion of the composite from water absorption can account for the apparent low wear during the first year. The line is the regression curve. Error bars represent one standard deviation.

$$
t_{\text {Clinic }}=\frac{V_{\text {Clinic }}}{V_{\text {Simulation }}} \times t_{\text {Simulation }}
$$

The simulation and clinical works are not the same unless $K_{\text {simulation }}$ equals $K_{\text {clinic }}$. The University of Minnesota artificial oral environment was designed to duplicate the biomechanics of human mastication so that the $K_{\mathrm{s}}$ would be similar, and Eq. (4) would be true.

Clinical data is often reported as depth of the wear facet rather than volume loss. Depth is not a good surrogate for volume. There are an infinite number of depths for a single volume due to changing areas. Comparing SureFil time conversions for depth and volume, it was found that the volume cycles/year were 2.3 to 2.7 times those of depth (Table III). This is partially explained by differences in contact wear areas. The average clinical wear area was $6.8 \mathrm{~mm}^{2}$ at Year 5 while the simulation areas were $2.8 \mathrm{~mm}^{2}(13 \mathrm{~N})$ and $3.9 \mathrm{~mm}^{2}(30 \mathrm{~N})$ at $1500 \mathrm{~K}$ cycles. Using the volume conversion rates (Table III), $1500 \mathrm{~K}$ cycles corresponds to 1.8 (13 $\mathrm{N})$ or $2.6(30 \mathrm{~N})$ years of clinical wear. The simulated areas correspond well to the clinical contact area of $3.3 \mathrm{~mm}^{2}$ for the Year 2 clinical wear area (Table I). If the depth conver-

\begin{tabular}{|c|c|c|c|c|c|}
\hline Wear Type & Parameter & Group & $r^{2}$ & Wear rate & Intercept \\
\hline Clinical Contact-free & Volume/Unit Area & $3+4$ & 0.54 & $0.007\left(\mathrm{~mm}^{3} / \mathrm{mm}^{2}\right) / \mathrm{Yr}^{* * *}$ & $-0.006 \mathrm{~mm}^{*}$ \\
\hline \multirow[t]{3}{*}{ Clinical Contact } & Volume & 4 & 0.52 & $0.129 \mathrm{~mm}^{3} / \mathrm{Yr}^{* * *}$ & $-0.050 \mathrm{~mm}^{3}$ \\
\hline & Depth & 4 & 0.64 & $0.012 \mathrm{~mm} / \mathrm{Yr}^{* * *}$ & $0.025 \mathrm{~mm}^{* * *}$ \\
\hline & Depth & $4 \mathrm{C}$ & 0.20 & $0.005 \mathrm{~mm} / \mathrm{Yr}^{* *}$ & $0.032 \mathrm{~mm}^{* * *}$ \\
\hline \multirow[t]{3}{*}{ Simulated Contact } & Volume & 5 & 0.58 & $0.016 \mathrm{~mm}^{3} / 10^{5}$ cycles $* * *$ & $0.007 \mathrm{~mm}^{3}$ \\
\hline & Depth & 5 & 0.56 & $0.004 \mathrm{~mm} / 10^{5}$ cycles $^{* * *}$ & $0.023 \mathrm{~mm}^{* * *}$ \\
\hline & Depth & 6 & 0.87 & $0.005 \mathrm{~mm} / 10^{5}$ cycles*** & $0.026 \mathrm{~mm}^{* * *}$ \\
\hline
\end{tabular}
sion rates are used, then $1500 \mathrm{~K}$ cycles corresponds to 5.0

TABLE II. Regression Analysis

\footnotetext{
${ }^{*} p<0.05$

** $p<0.01$;

$* * * p<0.001$
} 
TABLE III. In Vitro Cycles Equal to One Year of Clinical Wear

\begin{tabular}{llc}
\hline In Vivo & \multicolumn{1}{c}{ In Vitro } & $\begin{array}{c}1 \text { Year }= \\
\text { Cycles } \times 10^{5}\end{array}$ \\
\hline Group 4 depth & Group 5 depth 13 N & 3.0 \\
Group 4 depth & Group 6 depth 30 N & 2.5 \\
Group 4C corrected & Group 5 depth 13 N & 1.2 \\
$\quad \begin{array}{l}\text { depth } \\
\text { Group 4C corrected } \\
\quad \text { depth }\end{array}$ & Group 6 depth 30 N & 1.0 \\
Group 4 volume & Group 5 volume 13 N & 8.2 \\
Group 4 volume & Group 6 volume 30 N & 5.7 \\
Group 4C corrected & Group 5 volume 13 N & 4.7 \\
$\quad \begin{array}{l}\text { volume } \\
\text { Group 4C corrected } \\
\text { volume }\end{array}$ & Group 6 volume 30 N & 3.2 \\
\hline
\end{tabular}

$(13 \mathrm{~N})$ or $6.0(30 \mathrm{~N})$ clinical years and the simulated Year 5 wear areas (2.8 or $3.9 \mathrm{~mm}^{2}$, respectively) are much smaller than the Year 5 clinically measured area $\left(6.8 \mathrm{~mm}^{2}\right)$. If the calculations are repeated using the corrected clinical data and corresponding corrected conversion factors (Table III), both simulated volume and depth areas under estimate the Year 5 clinical area. Another contributing factor is the variation of natural chewing motion that causes a larger area of contact on the restoration. This variation does not occur in vitro. The volume measure accounts for differences in contact area; the depth measure does not. Thus, volume is the parameter of choice when measuring wear.

All regressions had offsets. These offsets are often attributed to an initial "wear in" period that results in an early increased wear rate, which is attributed to initial high spots that wear rapidly. ${ }^{18}$ Other possible contributors that apply specifically to composites are changes in composite hardness and volume that can occur during the first months intraorally. SureFil's hardness was shown to increase for six months when stored in artificial saliva. ${ }^{19}$ Over a 10 day period, SureFil had a volume expansion of $0.5 \%$ in artificial

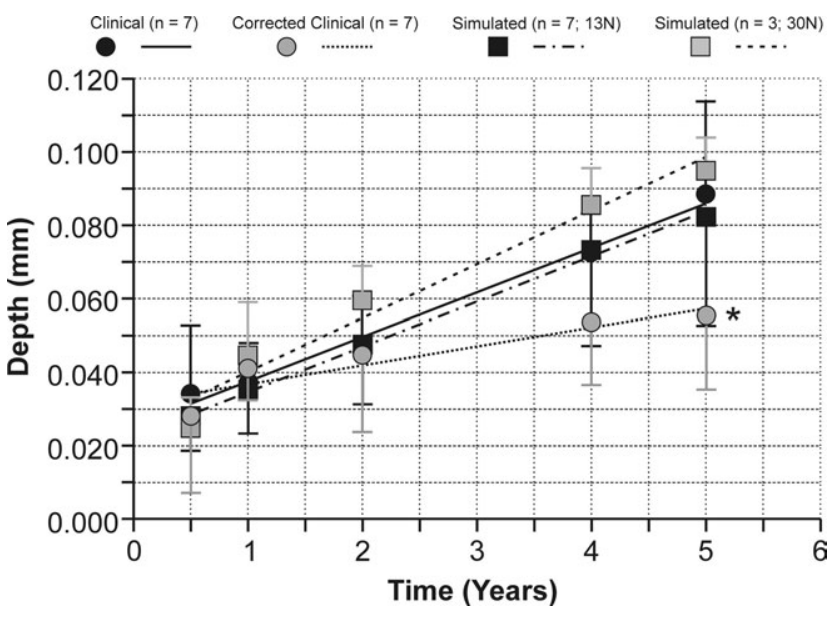

FIGURE 6. Clinical versus simulated contact wear mean depth. The corrected data points are the contact depth minus the contact-free depth. The error bars represent the standard deviations. The conversion factor was 300,000 cycles equals one year of clinical wear. * Significant with $p<0.05$.

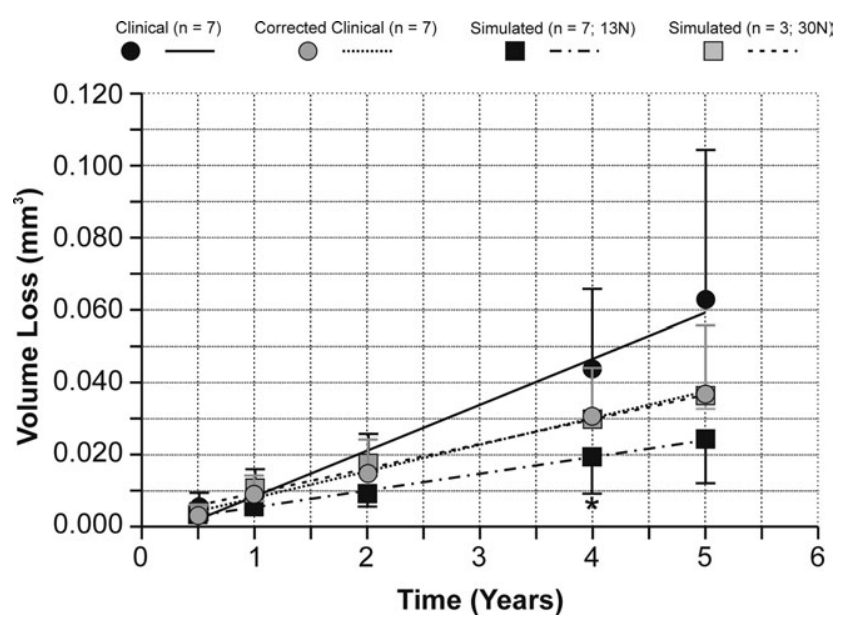

FIGURE 7. Clinical versus simulated contact wear mean volume loss. The corrected data points are the contact volume minus the contactfree volume for an area equal to that of the contact wear area. Regression lines for the corrected clinical data and the simulated data using a peak force of $30 \mathrm{~N}$ are nearly identical. The error bars represent the standard deviations. The conversion factor was 300,000 cycles equals one year of clinical wear. * Significant with $p<0.05$.

saliva. ${ }^{20}$ Effects of expansion could be seen in the noncontact wear as evidenced by the negative offset (Table II; Figure 5). These changes were not as obvious in the clinical or simulated contact wear groups; possibly due to the greater contact wear rates. Changes in hardness and expansion have greater effects on in vitro wear studies because wear is generated over days compared to years in clinical studies. Storing simulation samples in water or artificial saliva for two weeks prior to testing will minimize these effects.

Calibration is best done using multiple materials with different wear mechanisms. Powers et al. ${ }^{21}$ compared twobody abrasion of nine experimental composites to two-year clinical wear of the same composites. The in vitro test moved the material against 600 grit silicone carbide paper

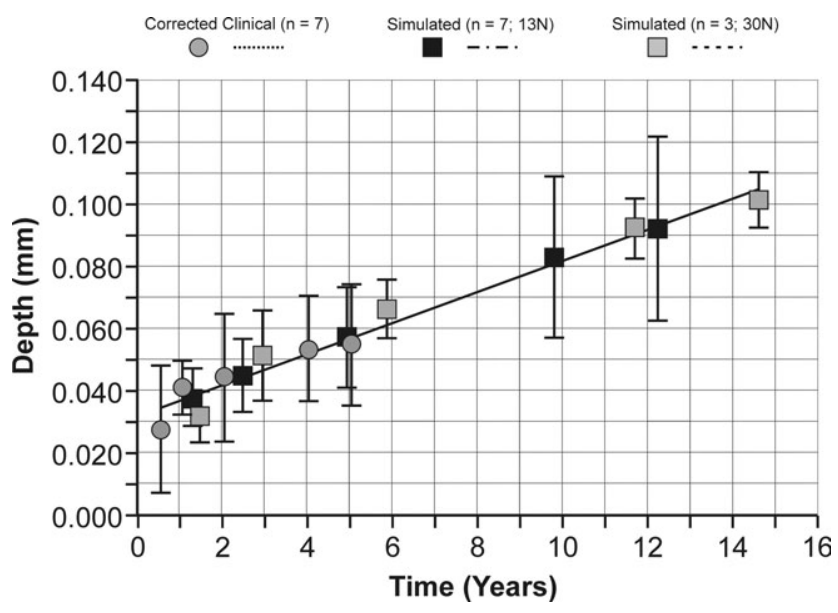

FIGURE 8. Corrected clinical versus simulated contact wear mean depth. The $15 \mathrm{~N}$ and $30 \mathrm{~N}$ simulated depths were converted from cycles to years using the conversion factors of Table III. All regression lines aligned. The error bars represent one standard deviation. 


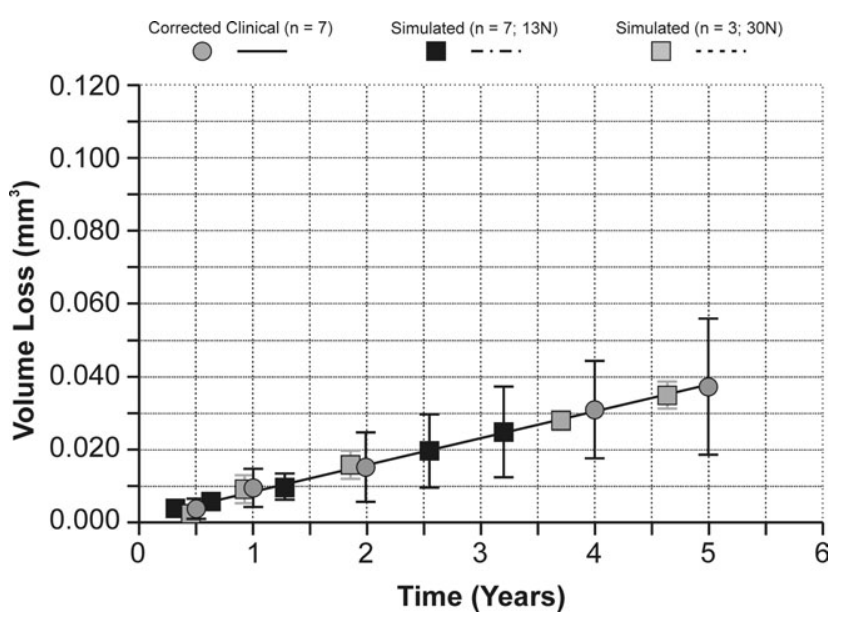

FIGURE 9. Corrected clinical versus simulated contact wear mean volume. The $15 \mathrm{~N}$ and $30 \mathrm{~N}$ simulated volumes were converted from cycles to years using the conversion factors of Table III. All regression lines aligned. The error bars represent one standard deviation.

under a $5 \mathrm{~N}$ load for a distance of $7110 \mathrm{~mm}$. Comparing in vivo ledge depths against in vitro composite total volume of material loss, they found a good correlation $\left(r^{2}=0.48\right)$ between the in vitro and in vivo data. Leinfelder and Suzuki, ${ }^{22}$ using a more sophisticated test method, compared in vivo and in vitro wear data for eleven materials including positive and negative controls. Their system used a PMMA bead slurry to provide three-body interactions and a $75 \mathrm{~N}$ impact force followed by rotational sliding. They also found a high correlation $\left(r^{2}=0.80\right)$ between the 3 year in vivo and the 400,000 cycle in vitro data when comparing in vivo ledge depths to in vitro depths.

The previous methods used clinical data measured at a single time point to determine material wear rates. This over estimates the actual wear rate because it assumes a constant wear rate starting from zero wear at time zero. Barkmeier et al., ${ }^{18}$ using the Leinfelder system, compared the in vivo and in vitro wear of P50 and $\mathrm{Z} 100$. Wear rates were defined as the slopes of the regression lines of the data. This method, which takes advantage of the linearity of the data and accounts for the early wear in, was adopted for this study. Clinical wear rates for P50 and Z100 were 38 and 27 microns/year. The clinical wear rate of SureFil was 12 microns/year (Group 4), which is about three times smaller. The in vitro P50, Z100, and SureFil results cannot meaningfully be compared because of unknown differences in the test systems. It is interesting to note that the average wear rate of the pooled P50 and Z100 in vitro data measured by maximum depth was 9.7 microns/year, which is about twice as large as the 4.9 microns/year for SureFil. If volume is the measure of wear, then the pooled wear rate for P50 and Z100 is $0.003 \mathrm{~mm}^{3}$ /year and that for SureFil is $0.023 \mathrm{~mm}^{3} /$ year, which is nearly an order of magnitude larger.

Initial calibration of the UMN simulation environment, which was done using amalgam, found good agreement between the simulated and clinical wear facet maximum depths using a $13 \mathrm{~N}$ peak force, $0.82 \mathrm{~mm}$ lateral movement, and a conversion rate of $2.5 \times 10^{5}$ cycles/year. $^{23}$ Using regression analysis, the conversion factor was $3 \times 10^{5}$ cycles/year, which agrees with the conversion factor found for SureFil with a $13 \mathrm{~N}$ force. This agreement in conversion factors is expected if the in vitro test reproduces the biophysical conditions of the oral cavity.

Sakaguchi et al. ${ }^{24}$ compared in vivo and in vitro wear for P10 composite over a one year trial using the UMN system with the same chewing parameters used in the amalgam study. They found excellent agreement in the in vitro $(0.059$ $\pm 0.008 \mathrm{~mm})$ and in vivo $(0.058 \pm 0.008 \mathrm{~mm})$ wear facet maximum depths at one year. The regression method conversion rate is $5 \times 10^{5}$ cycles/year, which is larger than that found for amalgam and SureFil. Because the clinical data for P10 only covers one year, the linear region may not have been reached.

The linear regression method forces data to the same regression line by adjusting the number of cycles equivalent to one year of clinical wear (Figures 8,9 ). The same result could be obtained by adjusting the force or contact path length. Increasing the force from $13 \mathrm{~N}$ to $30 \mathrm{~N}$ aligned the simulated volume and corrected clinical data (Figure 7). By adjusting simulation parameters, it is theoretically possible to find constant values for the force, contact distance, and number of cycles that will work for all materials. Under these conditions, the in vitro test system is truly calibrated. An obvious disadvantage of this method is the large amount of work involved.

Statistically, there was no difference between the in vitro and in vivo data except the in vitro depths at Year 5, which were significantly different from the clinical corrected depth, and the in vitro $13 \mathrm{~N}$ volume at Year 4, which was significantly different from the clinical volume. The fact that the in vitro values were not significantly different from the clinical values could be a result of the large variations in the data, the small number of specimens, or both.

It would have been advantageous to configure the simulated environment to produce three-body abrasion or erosion. Including a third body, as was done by Leinfelder and Suzuki, ${ }^{22}$ could produce abrasive wear; however, simulating erosion is more difficult. To "correct" the clinical contact wear for abrasion and erosion effects, the contactfree data from the same restoration was used. This correction assumed that abrasion and erosion effects were the same in both the contact and contact-free regions. The validity of this assumption needs further investigation.

Finally, compromises in conducting laboratory investigations after the start of a clinical trial must be made. The batches of material were not exactly the same. Restorative material used in the simulation was fresh and had not undergone any potential changes from absorption or long-term chemical changes that could have altered the materials properties. At some future date, it might be interesting to recover samples from both the clinical trial and the simulation for investigation of the chemical compositions. 


\section{CONCLUSIONS}

Within the limitations of this study, the following could be concluded:

1. Volume is the preferred parameter for measuring wear.

2. Under the conditions of this study, both the $13 \mathrm{~N}$ and 30 $\mathrm{N}$ peak forces provided good agreement between the simulated and clinical mean depths.

3. Under the same conditions, a peak force of $30 \mathrm{~N}$ provided the best agreement of the volume data for simulated and clinical contact wear.

\section{ACKNOWLEDGMENTS}

The authors wish to thank Mr. S Lunos of the University of Minnesota Biostatistical Design and Analysis Center for the statistical analysis. The clinical research trial conducted at the University of North Carolina involving SureFil was supported by Dentsply-Caulk.

\section{REFERENCES}

1. NIH/NIDCR. Increasing the Service Life of Dental Resin Composites (R01). RFA-DE-10-004, 2009.

2. Jandt KD, Sigusch BW. Future perspectives of resin-based dental materials. Dent Mater 2009;25:1001-1006.

3. Jokstad A, Bayne S, Blunck U, Tyas M, Wilson N. Quality of dental restorations. FDI Commission Project 2-95. Int Dent J 2001;51:117-158.

4. Loguercio AD, Reis A, Hernandez PAG, Macedo RP, Busato ALS. 3-Year clinical evaluation of posterior packable composite resin restorations. J Oral Rehabil 2006;33:144-151.

5. Poon ECM, Smales RJ, Yip KHK. Clinical evaluation of packable and conventional hybrid posterior resin-based composites: Results at 3.5 years. J Am Dent Assoc 2005;136:1533-1540.

6. Turkun LS, Turkun M, Ozata F. Clinical performance of a packable resin composite for a period of 3 years. Quintessence Int 2005;36:365-372.

7. Bayne SC, Wilder AD, Swift EJ Jr. Wear anomalies in 5-year clinical trial data for packable composite. J Dent Res 2005;84(Special Issue A):\#583.

8. Leinfelder KF, Taylor DF, Barkmier WW, Goldberg AJ. Quantitative wear measurement of posterior composite resins. Dent Mater 1986;2:198-201
9. DeLong R, Pintado MR, Douglas WH. Measurement of change in surface contour by computer graphics. Dent Mater 1985;1: 27-30.

10. Braem M, Lambrechts $P$, Van Doren V, Vanherle G. In vivo evaluation of four posterior composites: Quantitative wear measurements and clinical behavior. Dent Mater 1986;2:106-113.

11. DeLong $R$, Douglas WH. An artificial oral environment for testing dental materials. IEEE Trans Biomed Eng 1991;38:339-345.

12. Ahlgren J, OWall B. Muscular activity and chewing force: A polygraphic study of human mandibular movements. Arch Oral Biol 1970;15:271-280.

13. DeLong R, Heinzen M, Hodges JS, Ko CC, Douglas WH. Accuracy of a system for creating 3D computer models of dental arches. J Dent Res 2003;82:438-442.

14. Archard JF. Contact and rubbing of flat surfaces. J Appl Phys 1953;24:981-988.

15. Youssef RE, Throckmorton GS, Ellis E III, Sinn DP. Comparison of habitual masticatory patterns in men and women using a custom computer program. J Prosthet Dent 1997;78:179-186.

16. DeLong R, Pintado MR, Douglas WH. The wear of enamel opposing shaded ceramic restorative materials: An in vitro study. J Prosthet Dent 1992;68:42-48.

17. Peters MCRB, DeLong R, Pintado MR, Pallesen U, Quist V, Douglas WH. Comparison of two measurement techniques for clinical wear. J Dent 1999;27:479-485.

18. Barkmeier WW, Latta MA, Erickson RL, Lambrechts P. Comparison of laboratory and clinical wear rates of resin composites. Quintessence Int 2004;35:269-274.

19. Saygili G, Sahmali S, Figen Demirel F. Changes in the mechanical properties of tooth-colored direct restorative materials in relation to time. Polym Adv Technol 2003;14:616-622.

20. Marin N, Jedynakiewicz NM, Fisher AC. Hygroscopic expansion and solubility of composite restoratives. Dent Mater 2003;19: 77-86.

21. Powers JM, Ryan MD, Hosking DJ, Goldberg AJ. Comparison of in vitro and in vivo wear of composites. J Dent Res 1983;62: 1089-1091.

22. Leinfelder KF, Suzuki S. In vitro wear device for determining posterior composite wear. J Am Dent Assoc 1999;130:1347-1353.

23. DeLong $R$, Sakaguchi RL, Douglas $W H$, Pintado MR. The wear of dental amalgam in an artificial mouth: A clinical correlation. Dent Mater 1985;1:238-242.

24. Sakaguchi RL, Douglas $W H$, DeLong $R$, Pintado MR. The wear of a posterior composite in an artificial mouth: A clinical correlation. Dent Mater 1986;2:235-240. 